\title{
Improved Vector Velocity Estimation using Directional Transverse Oscillation
}

\author{
Jensen, Jørgen Arendt
}

Published in:

Proceedings of 2015 IEEE International Ultrasonics Symposium

Link to article, DOI:

10.1109/ULTSYM.2015.0011

Publication date:

2015

Document Version

Peer reviewed version

Link back to DTU Orbit

Citation (APA):

Jensen, J. A. (2015). Improved Vector Velocity Estimation using Directional Transverse Oscillation. In Proceedings of 2015 IEEE International Ultrasonics Symposium IEEE.

https://doi.org/10.1109/ULTSYM.2015.0011

\section{General rights}

Copyright and moral rights for the publications made accessible in the public portal are retained by the authors and/or other copyright owners and it is a condition of accessing publications that users recognise and abide by the legal requirements associated with these rights.

- Users may download and print one copy of any publication from the public portal for the purpose of private study or research.

- You may not further distribute the material or use it for any profit-making activity or commercial gain

- You may freely distribute the URL identifying the publication in the public portal

If you believe that this document breaches copyright please contact us providing details, and we will remove access to the work immediately and investigate your claim. 


\title{
Improved Vector Velocity Estimation using Directional Transverse Oscillation
}

\author{
Jørgen Arendt Jensen \\ Center for Fast Ultrasound Imaging, Department of Electrical Engineering, \\ Technical University of Denmark, DK-2800 Lyngby, Denmark
}

\begin{abstract}
A method for estimating vector velocities using transverse oscillation (TO) combined with directional beamforming is presented. Directional Transverse Oscillation (DTO) is selfcalibrating, which increase the estimation accuracy and finds the lateral oscillation period automatically. A normal focused field is emitted and the received signals are beamformed in the lateral direction transverse to the ultrasound beam. A lateral oscillation is obtained by having a receive apodization waveform with two separate peaks. The IQ data are obtained by making a Hilbert transform of the directional signal, and a modified TO estimator can be used to find both the lateral and axial velocity. The approach is self-calibrating as the lateral oscillation period directly is estimated from the directional signal through a Fourier transform. The approach was implemented on the SARUS scanner using a BK Medical 8820e transducer with a focal point at $105.6 \mathrm{~mm}(\mathrm{~F} \#=5)$ for Vector Flow Imaging (VFI). A $6 \mathrm{~mm}$ radius tube in a circulating flow rig was scanned and the parabolic volume flow of $112.7 \mathrm{l} / \mathrm{h}$ (peak velocity 0.55 $\mathrm{m} / \mathrm{s}$ ) measured by a Danfoss Magnetic flow meter for reference. Velocity estimates for DTO are found for $\mathbf{3 2}$ emissions at a 90 degrees beam-to-flow angle at a vessel depth of $30 \mathrm{~mm}$. The standard deviation (SD) drops from $9.14 \%$ for TO to $5.4 \%$, when using DTO. The bias is $\mathbf{- 5 . 0 5 \%}$ and the angle is found within +/3.93 degrees. At $70 \mathrm{~mm}$ a relative SD of $7 \%$ is obtained, the bias is $-1.74 \%$, and the angle is found within +/- 2.6 degrees showing a low bias across depths.
\end{abstract}

\section{INTRODUCTION}

When investigating human hemodynamics, it is important to find both the velocity magnitude and direction. The velocity vector in a plane can be estimated using the transverse oscillation (TO) method described in [1], [2], [3], [4]. A similar approach has also been suggested by Anderson [5] and studied by Sumi [6] and Liebgott et al. [7], [8], [9].

In TO vector flow imaging (VFI) two beams are formed during receive processing. The two beams have to be phased shifted a quarter of the lateral wavelength. This wavelength depends on the emit focus, receive apodization function, and the interrogation depth. The TO wavelength be predicted from [1], [10]:

$$
\lambda_{x}=\frac{2 \lambda d}{P_{d}}=\frac{2 \lambda d}{N_{d} P_{i}}
$$

where $\lambda$ is the normal axial wavelength, $d$ is the depth, and $P_{d}$ is the distance between the two peaks in the apodization function. The transducer pitch is $P_{i}$ and the number of elements between the peaks is $N_{d}$. The lateral wavelength depends on depth and therefore has to be calculated for every depth to ensure an unbiased and accurate result. The equation is also

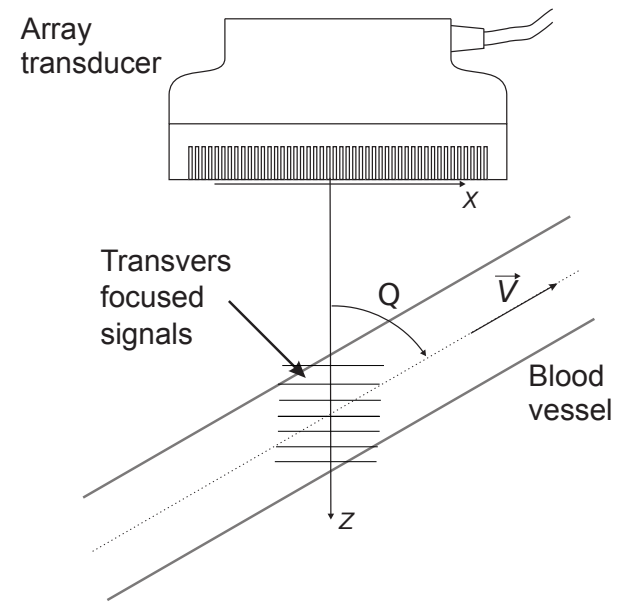

Fig. 1. Focusing of the received signals to generate a signal, which is transverse to the ultrasound direction.

only valid in the far-field or at the focus, and for a pulsed field this can introduce a significant bias and optimization has to be employed. This complicates the implementation of the approach [11].

A new method for beamforming transverse to the ultrasound direction is therefore suggested. The beams can be used for velocity estimation, and the lateral oscillation period or wavelength can also be estimated from this data making the method self-calibrating without the need for pre-calculation of the lateral wavelength.

\section{DiRECTIONAL TRANSVERSE OSCILLATION APPROACH}

The suggestion is to beamform a received signal in the lateral direction as shown in Fig. 1. A normal focused field is emitted, and the signals are received on all transducer elements. A beam $x(n, i)$ is then focused at the depth of interest in a direction transverse to the ultrasound propagation direction. Here $n$ is the sample number along the directional line, and $i$ is the pulse emission number. Bonnefous [12] suggested focusing a transverse beam with normal focusing and focusing along the flow direction was suggested by [13], [14], [15]. The key idea here is, however, to introduce a lateral oscillation and not have to focus along the flow.

A lateral oscillation is obtained, if a receive apodization waveform is employed as shown in Fig. 2 with two peaks separated by a distance. Two peaks in the apodization can 

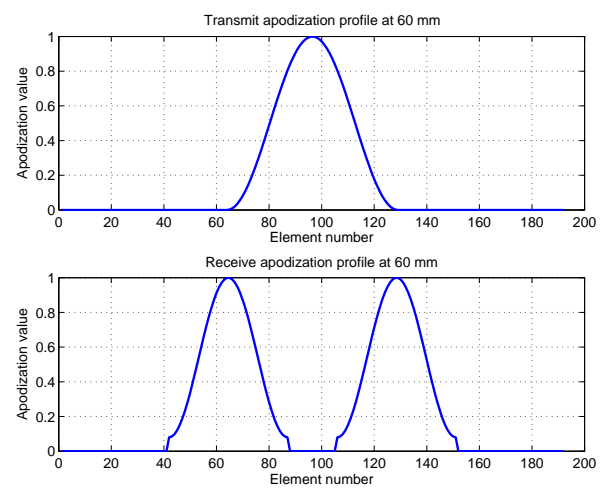

Fig. 2. The top graph shows the apodization employed during transmit, and the graph at the bottom shows a typical receive apodization function employed in TO imaging.

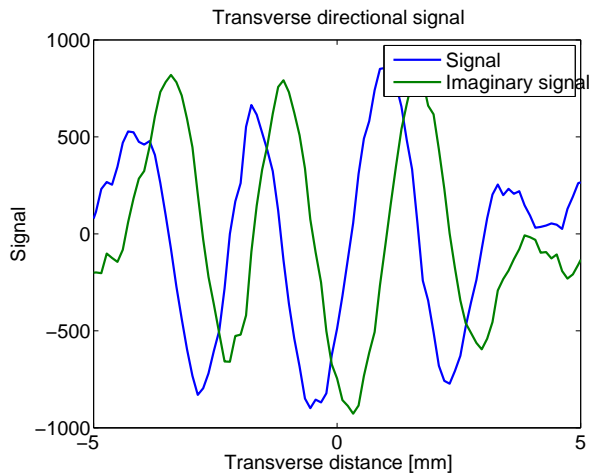

Fig. 3. Directional signal obtained from the focusing. The blue curve is the directional signal and the green curve is the Hilbert transform of the directional signal.

also be employed for the transmit beam to generate a lateral oscillation with a shorter wavelength.

A signal as a function of lateral distance is then obtained. It is shown as the blue curve in Fig. 3. To perform the velocity estimation, the quadrature signal is also needed [3]. This can be obtained by performing a Hilbert transform of the directional signal:

$$
y(n, i)=\mathscr{H}\{x(n, i)\},
$$

where $x(n, i)$ is the received signal as a function of emission number $i$, lateral sample number $n$, and $\mathscr{H}$ denotes Hilbert transform. It is shown as the green curve in Fig. 3. This yields a combined complex signal with a one-sided spectrum, which directly can be used by the estimator. The frequency content of one directional signal yields the lateral oscillation frequency, as the spectrum of the RF ultrasound signal yields the ultrasound frequency.

\section{Velocity ESTIMATION}

The estimator developed here is based on the vector velocity estimator for TO imaging described in [3], [4] and modified to include averaging across all samples in the directional signal. A complex signal is formed from the beamformed directional signal and its Hilbert transform as

$$
r_{s q}(n, i)=x(n, i)+j y(n, i) .
$$

The received element signals from the transducer is then Hilbert transformed in the temporal direction, and a new directional beamformed signal formed at the same depth for these data. This gives the signal $r_{s q h}(n, i)$. Two new signals are then formed from:

$$
\begin{aligned}
& r_{1}(n, i)=r_{s q}(n, i)+j r_{s q h}(n, i) \\
& r_{2}(n, i)=r_{s q}(n, i)-j r_{s q h}(n, i) .
\end{aligned}
$$

A simple model for the received signal is then [16], [3]:

$$
\begin{aligned}
r_{1}(n, i)= & k \cdot \exp \left(j 2 \pi \left(\left(\frac{2 v_{z}}{c} f_{0} i T_{p r f}-f_{0}\left(t-\frac{2 d}{c}\right)\right)\right.\right. \\
& \left.+\left(\frac{v_{x}}{\lambda_{x}} i T_{p r f}-n \Delta x / \lambda_{x}\right)\right)
\end{aligned}
$$

when assuming monochromatic signals. Here $v_{z}$ is the axial velocity, $v_{x}$ lateral velocity, $c$ speed of sound, $f_{0}$ transducer frequency, $\Delta x$ is the sampling interval along the lateral signal, $k$ is a constant, and $T_{p r f}$ is the time between pulse emissions. The interrogation depth is $d$ and the two frequencies received from the axial and lateral motions are given by:

$$
f_{p}=\frac{2 v_{z}}{c} f_{0}=\frac{2 v_{z}}{\lambda}, \quad f_{x}=\frac{v_{x}}{\lambda_{x}} .
$$

The changes in phase as a function of emission number for the two signals $r_{1}(n, i)$ and $r_{2}(n, i)$ are [3]:

$$
\begin{aligned}
d \Theta_{1} & =2 \pi T_{p r f}\left(f_{x}+f_{p}\right) \\
d \Theta_{2} & =2 \pi T_{p r f}\left(f_{x}-f_{p}\right) .
\end{aligned}
$$

Adding the two phase changes gives

$$
d \Theta_{1}+d \Theta_{2}=2 \pi 2 T_{p r f} f_{x}=4 \pi T_{p r f} \frac{v_{x}}{\lambda_{x}}
$$

and subtracting them gives

$$
d \Theta_{1}-d \Theta_{2}=2 \pi 2 T_{p r f} f_{p}=4 \pi T_{p r f} \frac{2 v_{z}}{\lambda} .
$$

The transverse velocity can, thus, be found directly from:

$$
v_{x}=\frac{\left(d \Theta_{1}+d \Theta_{2}\right) \lambda_{x}}{2 \pi 2 T_{p r f}}
$$

and the axial velocity from

$$
v_{z}=\frac{\left(d \Theta_{1}-d \Theta_{2}\right) \lambda}{2 \pi 4 T_{p r f}} .
$$

For a complex signal the phase change is determined by [16], [17]

$$
\begin{aligned}
d \hat{\Theta} & =\arctan \left(\frac{\sum_{i=0}^{N-1} y(i) x(i-1)-y(i-1) x(i)}{\sum_{i=0}^{N-1} x(i) x(i-1)+y(i) y(i-1)}\right) \\
& =\arctan \left(\frac{\mathfrak{I}\{R(1)\}}{\mathfrak{R}\{R(1)\}}\right),
\end{aligned}
$$

using the estimated complex autocorrelation of the signal

$$
\hat{R}(m)=\frac{1}{N-m} \sum_{i=0}^{N-m} r^{*}(i) r(i+m),
$$


where $\mathfrak{I}\{R(1)\}$ denotes the imaginary part of the complex autocorrelation and $\Re\{R(1)\}$ the real part both at a lag of 1 .

For the directional signals the autocorrelation function estimates are:

$$
\hat{R}_{1}(1)=\frac{1}{N N_{s}} \sum_{i=0}^{N-2} \sum_{n=0}^{N_{s}-1} r_{1}^{*}(n, i) r_{1}(n, i+1),
$$

and

$$
\hat{R}_{2}(1)=\frac{1}{N N_{s}} \sum_{i=0}^{N-2} \sum_{n=0}^{N_{s}-1} r_{2}^{*}(n, i) r_{2}(n, i+1) .
$$

The autocorrelation estimates are, thus, averaged over the number of emissions $N$ and the number of samples in the directional lines $N_{s}$. This reduces the noise and improves on estimation accuracy.

The velocity estimators for the two velocity components are then:

$$
v_{x}=\frac{\lambda_{x}}{2 \pi 2 T_{p r f}} \arctan \left(\frac{\mathfrak{I}\left\{R_{1}(1)\right\} \Re\left\{R_{2}(1)\right\}+\mathfrak{I}\left\{R_{2}(1)\right\} \mathfrak{R}\left\{R_{1}(1)\right\}}{\mathfrak{R}\left\{R_{1}(1)\right\} \Re\left\{R_{2}(1)\right\}-\mathfrak{I}\left\{R_{1}(1)\right\} \mathfrak{I}\left\{R_{2}(1)\right\}}\right)_{(14)}
$$

and

$v_{z}=\frac{\lambda}{2 \pi 4 T_{p r f}} \arctan \left(\frac{\mathfrak{I}\left\{R_{1}(1)\right\} \mathfrak{R}\left\{R_{2}(1)\right\}-\mathfrak{I}\left\{R_{2}(1)\right\} \mathfrak{R}\left\{R_{1}(1)\right\}}{\mathfrak{R}\left\{R_{1}(1)\right\} \mathfrak{R}\left\{R_{2}(1)\right\}+\mathfrak{I}\left\{R_{1}(1)\right\} \mathfrak{I}\left\{R_{2}(1)\right\}}\right)$,

when using the derivation from [3] to combine the two phase shift estimates into one equation. Here $\mathfrak{I}$ denotes imaginary part and $\Re$ real part.

The lateral wavelength needed in the estimator can be calculated by:

$$
1 / \lambda_{x}=f_{x}=\frac{\sum_{m=-N / 2}^{N / 2} \frac{m}{N \Delta x} R_{s q}(m, i)}{\sum_{m=-N / 2}^{N / 2} R_{s q}(m, i)}
$$

where $R_{s q}(m, i)$ is the Fourier transform of the complex directional signal $r_{s q}(n, i)=x(n, i)+j y(n, i)$ along the sample direction $n$. This makes the approach self calibrating as in [18] for axial velocity estimation. The lateral wavelength $\lambda_{x}$ can be calculated for the different depths and the estimator automatically yields an unbiased estimate of the transverse velocity. This frequency estimate can also be improved by averaging the estimator over all emissions as

$$
f_{x}=\frac{1}{N} \sum_{i=0}^{N-1} \frac{\sum_{m=-N / 2}^{N / 2} \frac{m}{N \Delta x} R_{s q}(m, i)}{\sum_{m=-N / 2}^{N / 2} R_{s q}(m, i)} .
$$

The beamforming can be performed for a normal focused emission, for synthetic aperture flow imaging, and for plane wave imaging. The transmit and receive apodization function can be changed as a function of depth to obtain the highest possible $f_{x}$. In synthetic aperture and plane wave imaging the combined transmit apodization function can also be manipulated to further increase the lateral oscillation frequency.

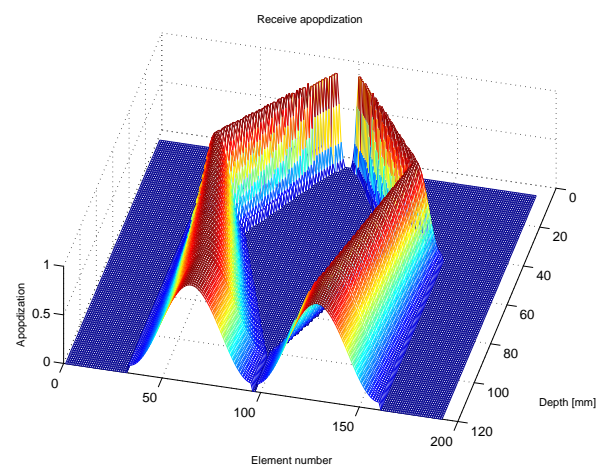

Fig. 4. Dynamic apodization profiles as a function of depth for the receive processing.

\section{Performance}

The velocity estimation approach has been implemented on the SARUS experimental scanner [19]. A BK Medical 8820 e convex array transducer was employed and vector flow imaging (VFI) in a single direction was interleaved with a B-mode image. An active aperture of 64 elements was used during transmit for both sequences. The focal point was at $42 \mathrm{~mm}(F \#=2)$ for the B-mode and $105.6 \mathrm{~mm}(F \#=5)$ for VFI. The transducer has 192 elements with $\lambda$ pitch and the B-mode image consisted of 129 lines. A $6 \mathrm{~mm}$ radius tube in a circulating flow rig was scanned and the volume flow was measured by a Danfoss Magnetic flow meter for reference. The volume flow was $112.7 \mathrm{l} / \mathrm{h}$ corresponding to a peak velocity in the vessel of $0.55 \mathrm{~m} / \mathrm{s}$. The pulse repetition frequency was $4 \mathrm{kHz}$ and the beam-to-flow angle is 90 degrees.

The beamformation employed a double Hamming apodization on the receiving aperture and the F-number in receive was 2 . The receiving aperture was expanded as a function of depth to maintain a constant F-number and thereby a constant $\lambda_{x}$. The resulting apodization profiles are shown in Fig. 4.

An example of velocity estimation for the traditional TO approach is shown in Fig. 5 and the corresponding estimates for DTO is shown in Fig. 6 when using 32 emissions. The standard deviation on the estimates drops from $9.14 \%$ to $5.4 \%$ when using the new approach.

The self-calibrating feature of the approach is shown in Fig. 7, where data from a vessel at $70 \mathrm{~mm}$ is used. The same beamforming scheme is used and a relative standard deviation of $7 \%$ is obtained with a bias of $-1.7 \%$ and the angle is found within 2.6 degrees.

\section{Discussion AND CONCLUSION}

A new method for making TO vector flow estimation selcalibrating has been suggested. The techniques find the lateral oscillation period from the actual measured data and uses this in the velocity estimation. This makes it possible to have any apodization waveform without having to predict the lateral oscillation period, and this maintains a low bias of the estimates for different depths. The approach also makes it possible to average over the lateral signal to decrease variance. Results from a circulating flow rig showed a decrease of 

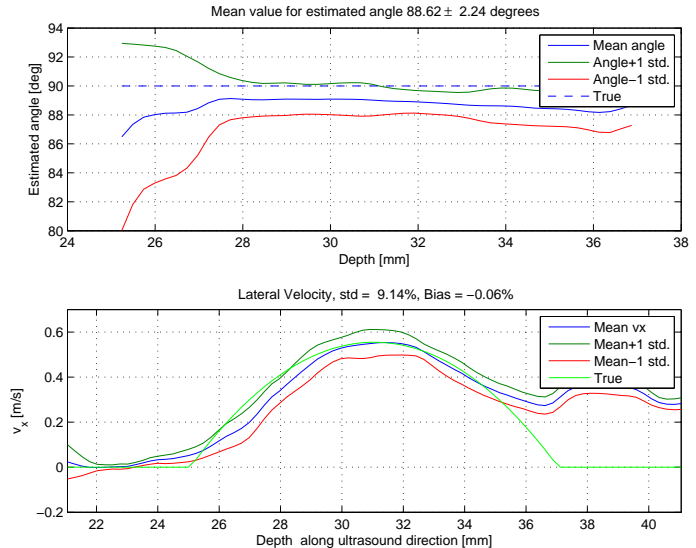

Fig. 5. Mean of velocity profiles at a 90 degrees beam to flow angle with 32 emissions using the traditional TO focusing approach optimized for this depth. The top figure shows the estimated angle and the bottom figure shows the mean of the 40 estimated profiles \pm one standard deviation. The vessel is placed $3 \mathrm{~cm}$ from the transducer.
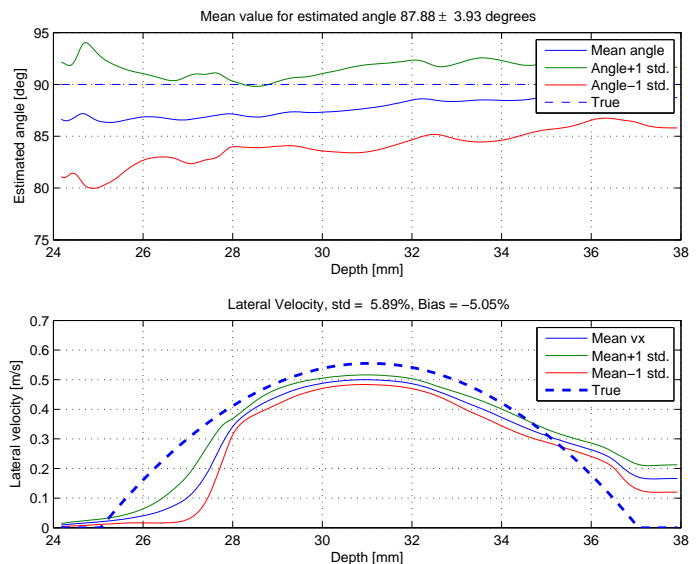

Fig. 6. Mean of velocity profiles at a 90 degrees beam to flow angle with 32 emissions using the new directional TO focusing approach. The top figure shows the estimated angle and the bottom figure shows the mean of the 40 estimated profiles \pm one standard deviation. The vessel is placed $3 \mathrm{~cm}$ from the transducer.
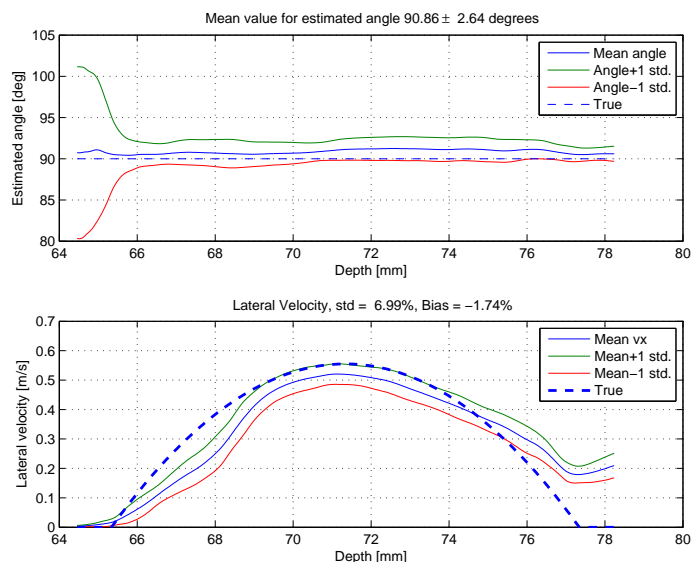

Fig. 7. Mean of velocity profiles at a 90 degrees beam to flow angle with 32 emissions using the new directional TO focusing approach. The top figure shows the estimated angle and the bottom figure shows the mean of the 40 estimated profiles \pm one standard deviation. The vessel is placed $7 \mathrm{~cm}$ from the transducer. standard deviation at $30 \mathrm{~mm}$ from $9.14 \%$ to $5.4 \%$ and the bias could be maintained below $5 \%$ for an interrogation depth from 30 to $70 \mathrm{~mm}$.

\section{ACKNOWLEDGEMENT}

This work was supported by grant 82-2012-4 from the Danish Advanced Technology Foundation and by B-K Ultrasound Aps.

\section{REFERENCES}

[1] J. A. Jensen and P. Munk, "A new method for estimation of velocity vectors," IEEE Trans. Ultrason., Ferroelec., Freq. Contr., vol. 45, pp. 837-851, 1998.

[2] J. A. Jensen, "Apparatus and method for determining movements and velocities of moving objects," International patent PCT/DK97/00287, 1996.

[3] - "A new estimator for vector velocity estimation," IEEE Trans. Ultrason., Ferroelec., Freq. Contr., vol. 48, no. 4, pp. 886-894, 2001.

[4] — "Estimation of vector velocity," International patent PCT/DK00/00243, 10 May 2000.

[5] M. E. Anderson, "Multi-dimensional velocity estimation with ultrasound using spatial quadrature," IEEE Trans. Ultrason., Ferroelec., Freq. Contr., vol. 45, pp. 852-861, 1998.

[6] C. Sumi, "Displacement vector measurement using instantaneous ultrasound signal phase - multidimensional autocorrelation and Doppler methods," IEEE Trans. Ultrason., Ferroelec., Freq. Contr., vol. 55, no. 1, pp. 24-43, 2008.

[7] H. Liebgott, J. Wilhjelm, J. A. Jensen, D. Vray, and P. Delachartre "PSF dedicated to estimation of displacement vectors for tissue elasticity imaging with ultrasound," IEEE Trans. Ultrason., Ferroelec., Freq. Contr., vol. 54, no. 4, pp. 746-756, 2007.

[8] H. Liebgott, A. Basarab, P. Gueth, C. Cachard, and P. Delachartre, "Lateral RF image synthesis using a synthetic aperture imaging technique." IEEE Trans. Ultrason., Ferroelec., Freq. Contr., vol. 55, no. 9, pp. 20972103, 2008

[9] A. Basarab, P. Gueth, H. Liebgott, and P. Delachartre, "Phase-based block matching applied to motion estimation with unconventional beamforming strategies," IEEE Trans. Ultrason., Ferroelec., Freq. Contr., vol. 56, no. 5, pp. 945-957, 2009.

[10] J. W. Goodman, Introduction to Fourier optics, 2nd ed. New York: McGraw Hill Inc., 1996.

[11] J. A. Jensen, "Optimization of transverse oscillating fields for vector velocity estimation with convex arrays," in Proc. IEEE Ultrason. Symp., July 2013, pp. 1753-1756.

[12] O. Bonnefous, "Measurement of the complete (3D) velocity vector of blood flows," in Proc. IEEE Ultrason. Symp., 1988, pp. 795-799.

[13] J. A. Jensen, "Vector velocity estimation using directional beamforming and cross-correlation, International patent," 1999.

[14] J. A. Jensen and S. I. Nikolov, "Directional synthetic aperture flow imaging," IEEE Trans. Ultrason., Ferroelec., Freq. Contr., vol. 51, pp. 1107-1118, 2004.

[15] J. A. Jensen, "Directional velocity estimation using focusing along the flow direction: I: Theory and simulation," IEEE Trans. Ultrason., Ferroelec., Freq. Contr., vol. 50, pp. 857-872, 2003.

[16] — Estimation of Blood Velocities Using Ultrasound: A Signal Processing Approach. New York: Cambridge University Press, 1996.

[17] C. Kasai, K. Namekawa, A. Koyano, and R. Omoto, "Real-Time TwoDimensional Blood Flow Imaging using an Autocorrelation Technique," IEEE Trans. Son. Ultrason., vol. 32, pp. 458-463, 1985.

[18] T. Loupas, J. T. Powers, and R. W. Gill, "An axial velocity estimator for ultrasound blood flow imaging, based on a full evaluation of the Doppler equation by means of a two-dimensional autocorrelation approach," IEEE Trans. Ultrason., Ferroelec., Freq. Contr., vol. 42, pp. 672-688, 1995.

[19] J. A. Jensen, H. Holten-Lund, R. T. Nilsson, M. Hansen, U. D. Larsen, R. P. Domsten, B. G. Tomov, M. B. Stuart, S. I. Nikolov, M. J. Pihl, Y. Du, J. H. Rasmussen, and M. F. Rasmussen, "SARUS: A synthetic aperture real-time ultrasound system," IEEE Trans. Ultrason., Ferroelec., Freq. Contr., vol. 60, no. 9, pp. 1838-1852, 2013. 